Wave Motion (in press)

\title{
Anisotropic elastic materials capable of a three-dimensional deformation (static or dynamic) with only one displacement component and uncoupling of all three displacement components
}

\author{
T. C. T. Ting ${ }^{1,2}$
}

\begin{abstract}
It is shown that there are anisotropic elastic materials that are capable of a nonuniform three-dimensional deformation with only one displacement component. For wave propagation the equation of motion can be cast in the form of the differential equation for acoustic waves. For elastostatics the equation of equilibrium reduces to Laplace's equation. The material can be monoclinic, orthotropic, tetragonal, hexagonal or cubic. There are also anisotropic elastic materials that uncouple all three displacement components. The governing equation for each of the uncoupled displacement can be cast in the form of the differential equation for acoustic waves in the case of dynamic or Laplace's equation in the case of static. The material can be orthotropic, tetragonal, hexagonal or cubic.
\end{abstract}

\section{Introduction}

For a two-dimensional deformation in which the displacement $\mathbf{u}$ depends on $x_{1}$ and $x_{2}$ only, it is shown in [1] that there are anisotropic elastic materials that can have only one displacement component other than the anti-plane displacement $u_{3}$. There are also anisotropic elastic materials for which all three displacement components are uncoupled. The purpose of this paper is to show that there are anisotropic materials that can have one displacement component that depends on $x_{1}, x_{2}$ and $x_{3}$. Without loss of generality we take $u_{3}$ as the one component displacement. The equation of motion for $u_{3}$ can be cast in the form of the differential equation for acoustic waves. For elastocstatics the equation of

\footnotetext{
1 Professor Emeritus of University of Illinois at Chicago.

2 Division of Mechanics and computation, Stanford University, Durand 262, Stanford, CA 94305 (tting@uic.edu)
} 
equilibrium reduces to Laplace's equation. The material can be any of the nine symmetry groups except triclinic, trigonal and isotropic. There are also anisotropic elastic materials that uncouple all three displacement components in threedimensional deformation, static or dynamic. The equation of motion for each uncoupled displacement can be cast in the form of the differential equation for acoustic waves. For elastostatics each equation is Laplace's equation. The material can be any of the nine symmetry groups except triclinic, monoclinic, trigonal and isotropic.

\section{Basic equations}

We consider wave propagation in a homogeneous linear anisotropic elastic medium. In a fixed rectangular coordinate system $x_{i}(i=1,2,3)$, the equation of motion is

$$
\sigma_{i j, j}=\rho \ddot{u}_{i}
$$

where $\sigma_{i j}$ is the stress, $u_{i}$ is the displacement, $\rho$ is mass density, the dot denotes differentiation with time $t$ and a comma denotes differentiation with $x_{i}$. The stressstrain relation is

$$
\begin{gathered}
\sigma_{i j}=C_{i j k s} u_{k, s}, \\
C_{i j k s}=C_{j i k s}=C_{k s i j}=C_{i j s k},
\end{gathered}
$$

in which $C_{i j k s}$ is the elastic stiffness. The $C_{i j k s}$ is positive definite and possesses the full symmetry shown in (2.3). The third equality in (2.3) is redundant because the first two imply the third [2]. Substitution of (2.2) into (2.1) leads to

$$
C_{i j k s} u_{k, s j}=\rho \ddot{u_{1}} \text {. }
$$

Consider a deformation that consists of only one displacement component. Without loss of generality let

$$
u_{1}=u_{2}=0, \quad u_{3}=W(\mathbf{x}, t)
$$

The equations of motion (2.4) for $i=1,2$ are trivially satisfied if 


$$
\begin{gathered}
C_{14}=C_{15}=C_{24}=C_{25}=C_{34}=C_{35}=C_{46}=C_{56}=0, \\
C_{36}+C_{45}=C_{13}+C_{55}=C_{23}+C_{44}=0,
\end{gathered}
$$

where $C_{\alpha \beta}$ is the contracted notation for $C_{i j k s}$. We will set

$$
C_{45}=0 \text {. }
$$

If $C_{45} \neq 0$, we rotate the coordinate system about the $x_{3}$-axis an angle $\psi$ given by

$$
\tan 2 \psi=\frac{2 C_{45}}{C_{55}-C_{44}} .
$$

The $C_{45}$ referred to the rotated coordinate system vanishes. The equations of motion (2.4) for $i=3$ reduces to

$$
C_{55} W,{ }_{11}+C_{44} W,{ }_{22}+C_{33} W,{ }_{33}=\rho W^{W} .
$$

The $6 \times 6$ elastic stiffness matrix $C_{\alpha \beta}$ that satisfies $(2.6 \mathrm{a}, \mathrm{b}, \mathrm{c})$ has the expression

$$
\mathbf{C}=\left[\begin{array}{cccccc}
C_{11} & C_{12} & -C_{55} & 0 & 0 & C_{16} \\
& C_{22} & -C_{44} & 0 & 0 & C_{26} \\
& & C_{33} & 0 & 0 & 0 \\
& & C_{44} & 0 & 0 \\
& & & & C_{55} & 0 \\
& & & & & C_{66}
\end{array}\right] .
$$

Only the upper triangle is shown because the matrix $\mathbf{C}$ is symmetric.

\section{Positive definiteness of the matrix $C$}

For the strain energy density to be positive, it is necessary and sufficient that the $6 \times 6$ matrix $C$ shown in (2.9) be positive definite. If we let

$$
C_{44}>0, C_{55}>0 \text {, }
$$

all we need is that the $4 \times 4$ matrix

$$
\hat{\mathbf{C}}=\left[\begin{array}{cccc}
C_{11} & C_{12} & -C_{55} & C_{16} \\
& C_{22} & -C_{44} & C_{26} \\
& & C_{33} & 0 \\
& & & C_{66}
\end{array}\right]
$$


be positive definite. A matrix is positive definite if all its leading principal minors are positive and non-zero. It is not difficult to show that one can choose $C_{11}, C_{22}$, $C_{33}$ and $C_{66}$, in that order, such that the leading principal minors of the $4 \times 4$ matrix in (3.2b) are positive and non-zero [3].

The material represented by (2.9) is monoclinic with the symmetry plane at $x_{3}=0$. It is orthotropic when

$$
C_{16}=C_{26}=0 \text {. }
$$

If (3.3) and

$$
C_{11}=C_{22}, \quad C_{44}=C_{55}
$$

hold, the material is tetragonal. When (3.3), (3.4) and

$$
C_{66}=\frac{1}{2}\left(C_{11}-C_{12}\right)
$$

hold, it is hexagonal. The material is cubic if (3.3) and

$$
C_{11}=C_{22}=C_{33}, \quad C_{44}=C_{55}=C_{66}=-C_{12}
$$

hold. Equation (2.9) cannot represent an isotropic material because the matrix $\mathbf{C}$ would be positive semi-definite.

\section{The stresses and strains}

With the displacement given by (2.5), the strains $\varepsilon_{i j}$ are

$$
\begin{gathered}
\varepsilon_{11}=\varepsilon_{22}=\varepsilon_{12}=0, \\
2 \varepsilon_{13}=W_{, 1}, \quad 2 \varepsilon_{23}=W_{, 2}, \quad \varepsilon_{33}=W_{, 3} .
\end{gathered}
$$

The stresses obtained from (2.2) and (2.9) are

$$
\begin{gathered}
\sigma_{11}=-C_{55} W, 3, \quad \sigma_{22}=-C_{44} W, 3, \quad \sigma_{33}=C_{33} W, 3, \\
\sigma_{12}=0, \quad \sigma_{13}=C_{55} W, \quad \sigma_{23}=C_{44} W, 2 .
\end{gathered}
$$


It is clear from the differential equation (2.5) and the stresses given in (4.2) that the solution depends on $C_{33}, C_{44}$ and $C_{55}$ only. They do not depend on $C_{11}, C_{12}, C_{22}$, $C_{16}, C_{26}$ and $C_{66}$. Thus the solutions for any two materials are identical when the elastic constants $C_{33}, C_{44}$ and $C_{55}$ in the two materials are the same. If $C_{33}=C_{44}=C_{55}$, the solution depends on one elastic constant.

\section{One-displacement motion}

Let

$$
\xi_{1}=\gamma_{1} x_{1}, \quad \xi_{2}=\gamma_{2} x_{2}, \quad \xi_{3}=x_{3},
$$

where

$$
\gamma_{1}=\frac{C_{33}}{C_{55}}, \quad \gamma_{2}=\frac{C_{33}}{C_{44}} .
$$

The equation of motion (2.8) simplifies to

$$
\frac{\partial^{2} W}{\partial \xi_{1}^{2}}+\frac{\partial^{2} W}{\partial \xi_{2}^{2}}+\frac{\partial^{2} W}{\partial \xi_{3}^{2}}=\frac{1}{c^{2}} W^{\tilde{W}} .
$$

In the above,

$$
c^{2}=C_{33} / \rho .
$$

Equation (5.3) is a differential equation for acoustic waves whose solution has been extensively studied in the literature (see [4], for example).

\section{Elastostatic with one displacement component}

For elastostatics, the displacement $W$ does not depend on time $t$. Equation (5.3) reduces to

$$
\frac{\partial^{2} W}{\partial \xi_{1}^{2}}+\frac{\partial^{2} W}{\partial \xi_{2}^{2}}+\frac{\partial^{2} W}{\partial \xi_{3}^{2}}=0
$$

This is Laplace's equation. Again, its solution has been extensively studied in the literature (see [5], for example).

\section{Uncoupling of all three displacement components}


It is not difficult to show that if the $6 \times 6$ elastic stiffness matrix $C_{\alpha \beta}$ has the structure

$$
\mathbf{C}=\left[\begin{array}{cccccc}
C_{11} & C_{66} & -C_{55} & 0 & 0 & 0 \\
& C_{22} & -C_{44} & 0 & 0 & 0 \\
& & C_{33} & 0 & 0 & 0 \\
& & & C_{44} & 0 & 0 \\
& & & & C_{55} & 0 \\
& & & & & C_{66}
\end{array}\right\rfloor,
$$

the equation of motion (2.4) for $u_{1}=U, u_{2}=V$ and $u_{3}=\mathrm{W}$ reduces to

$$
\begin{aligned}
& C_{11} U_{, 11}+C_{66} U,{ }_{, 22}+C_{55} U_{, 33}=\rho \overleftrightarrow{\mathbb{H},} \\
& C_{66} V,,_{11}+C_{22} V,,_{22}+C_{44} V,{ }_{33}=\rho \hat{V}, \\
& C_{55} W,_{11}+C_{44} W,_{22}+C_{33} W,{ }_{33}=\rho W .
\end{aligned}
$$

They are completely uncoupled. The equations for $U$ and $V$ are similar to the equation for $W$. Hence they can be reduced to the equation for acoustic waves. For elastostatics, they can be reduced to Laplace's equation.

Chadwick and Norris [6] discovered the materials listed in (7.1) through their study of anisotropic elastic materials whose slowness surface is the union of aligned ellipsoids. The acoustic tensor is a diagonal matrix so that the three polarization vectors are along the $x_{1}$-axis, $x_{2}$-axis and $x_{3}$-axis. They did not mention that the three equations of motion are uncoupled, nor the fact that each uncoupled equation of motion can be cast in the form of the differential equation for acoustic waves.

\section{Remarks}

The materials described in (2.9) and (7.1) may not exist in real materials. With the advancement in technology on manufacturing composites, it maybe possible in the future to produce the material specified in (2.9) and (7.1).

It should be noted that, for a one-displacement solution to exist the initial and boundary values cannot be prescribed arbitrarily. 


\section{References}

[1] T. C. T. Ting, "Anisotropic elastic materials that uncouple all three displacement components, and existence of one-displacement Green's function", J. Elasticity 57, 133-155 (2000).

[2] T. C. T. Ting, Anisotropic Elasticity: Theory and Applications, Oxford University Press, Oxford (1996), 32.

[3] T. C. T. Ting, "Positive definitness of anisotropic elastic constants", Math. Mech. Solids 1, 301-314 (1996).

[4] R. Courant and D. Hilbert, Methods of Mathematical Physics, vol II, Interscience (Wiley) New York (1962).

[5] L. C. Evans, Partial Differential Equations, Providence: American Mathematical Society (1998).

[6] P. Chadwick and A. N. Norris, "Conditions under which the slowness surface of an anisotropic elastic material is the union of aligned ellipsoids”, Q. J. Mech. Appl. Math. 43, 589-603 (1990). 